17. J. L. Hoogland, Science 215, 1639 (1982).

18. J. L. Hoogland, The Black-tailed Prairie Dog: Social Life of a Burrowing Mammal (Univ. of Chicago Press, Chicago, 1995).

19. J. L. Hoogland, J. Mammal. 80, 243 (1999).

20. J. L. Hoogland, in Rodent Societies, ]. O. Wolff, P. W. Sherman, Eds. (Univ. of Chicago Press, Chicago, 2007), pp. 438-450.

21. Details about my materials and methods are available as supplementary materials on Science Online.

22. J. B. Silk, Philos. Trans. R. Soc. London Ser. B 362, 539 (2007).

23. J. C. Mitani, ]. Call, P. M. Kappeler, R. Palombit, ]. B. Silk, The Evolution of Primate Societies (Univ. of Chicago Press, Chicago, 2012).

24. ]. L. Hoogland, Behaviour 69, 1 (1979).

25. J. L. Hoogland, Behav. Ecol. Sociobiol. 63, 1621 (2009).

26. P. ]. Greenwood, Anim. Behav. 28, 1140 (1980).

27. P. M. Waser, W. T. Jones, Q. Rev. Biol. 58, 355 (1983).
28. W. D. Koenig, D. Van Vuren, P. N. Hooge, Trends Ecol. Evol. 11, 514 (1996).

29. C. M. Arnaud, F. S. Dobson, ]. O. Murie, Mol. Ecol. 21, 493 (2012).

30. K. B. Armitage, D. H. Van Vuren, A. Ozgul, M. K. Oli, Ecology 92, 218 (2011).

Acknowledgments: I thank ]. Hoogland for encouraging me to reexamine my information on dispersal; my 150+ research assistants over the 31 years of research (especially my four offspring); and D. Boesch, K. Fuller, R. Gardner, R. Morgan, and L. Pitelka of the University of the Maryland Center for Environmental Science (UMCES) for the opportunity for long-term comparative research. Financial support was provided by Colorado Parks and Wildlife, the Denver Zoological Foundation, Environmental Defense, the Eppley Foundation, the Harry Frank Guggenheim Foundation, the National Fish and Wildlife Foundation, the National Geographic Society,
NSF, Princeton University, the Ted Turner Foundation, UMCES, and the Universities of Michigan and Minnesota. For help with the manuscript, I thank R. Alexander, D. Blumstein, D. Bowler, C. Brown, ]. Clobert, T. H. Clutton-Brock, A. Davis-Robosky, F. S. Dobson, L. Handley, K. Holekamp, A. Hoogland, S. Keller, X. Lambin, M. Oli, P. Sherman, N. Solomon, and D. Van Vuren. Data for this report are archived as supplementary materials on Science Online.

\section{Supplementary Materials}

www.sciencemag.org/cgi/content/full/339/6124/1205/DC1 Materials and Methods

Fig. S1

Tables S1 and S2

References (31-38)

18 October 2012; accepted 23 January 2013

$10.1126 /$ science. 1231689

\title{
Gene Transfer from Bacteria and Archaea Facilitated Evolution of an Extremophilic Eukaryote
}

\author{
Gerald Schönknecht, ${ }^{1,2 *} \dagger$ Wei-Hua Chen, ${ }^{3,4} \dagger$ Chad M. Ternes, ${ }^{1} \dagger$ Guillaume G. Barbier, ${ }^{5} \dagger \ddagger$
} Roshan P. Shrestha, ${ }^{5} \dagger \S$ Mario Stanke, ${ }^{6}$ Andrea Bräutigam, ${ }^{2}$ Brett ]. Baker, ${ }^{7}$ jillian F. Banfield, ${ }^{8}$ R. Michael Garavito, ${ }^{9}$ Kevin Carr, ${ }^{10}$ Curtis Wilkerson, ${ }^{5,10}$ Stefan A. Rensing, ${ }^{11}||$ David Gagneul, ${ }^{12}$ Nicholas E. Dickenson, ${ }^{13}$ Christine Oesterhelt, ${ }^{14}$ Martin J. Lercher, ${ }^{3,15}$ Andreas P. M. Weber ${ }^{2,5,15 *}$

Some microbial eukaryotes, such as the extremophilic red alga Galdieria sulphuraria, live in hot, toxic metal-rich, acidic environments. To elucidate the underlying molecular mechanisms of adaptation, we sequenced the 13.7-megabase genome of $G$. sulphuraria. This alga shows an enormous metabolic flexibility, growing either photoautotrophically or heterotrophically on more than 50 carbon sources. Environmental adaptation seems to have been facilitated by horizontal gene transfer from various bacteria and archaea, often followed by gene family expansion. At least $5 \%$ of protein-coding genes of $G$. sulphuraria were probably acquired horizontally. These proteins are involved in ecologically important processes ranging from heavy-metal detoxification to glycerol uptake and metabolism. Thus, our findings show that a pan-domain gene pool has facilitated environmental adaptation in this unicellular eukaryote.

A lthough bacteria and archaea usually dominate extreme environments, hot and extremely acidic habitats are typically devoid of photosynthetic bacteria. Instead, eukaryotic unicellular red algae of the Cyanidiophyceae are the principal photosynthetic organisms in these ecological niches (1). Cyanidiophyceae can grow at $\mathrm{pH} 0$ to 4 and temperatures up to $56^{\circ} \mathrm{C}$, close to the upper temperature limit for eukaryotic life (2). Galdieria sulphuraria is a unique member of the Cyanidiophyceae, displaying high salt and metal tolerance and exhibiting extensive metabolic versatility $(3,4)$. G. sulphuraria naturally inhabits volcanic hot sulfur springs, solfatara soils, and anthropogenic hostile environments. In habitats with high concentrations of arsenic, aluminum, cadmium, mercury, and other toxic metals, G. sulphuraria frequently represents up to $90 \%$ of total biomass and almost all the eukaryotic biomass $(1,5)$.

To understand the molecular mechanisms underlying G. sulphuraria's extremophilic and metabolically flexible lifestyle (Fig. 1), we determined its genome sequence (13.7 Mb; table $\mathrm{S} 1$ )
(6). The only member of the Cyanidiophyceae for which a genome sequence was previously available, Cyanidioschyzon merolae (7), diverged from $G$. sulphuraria about 1 billion years ago, which approximates the evolutionary distance between fruit flies and humans (see fig. S1 and supplementary materials). C. merolae maintains a strictly photoautotrophic lifestyle and does not tolerate high salt or metal concentrations; it differs markedly from $G$. sulphuraria in ecology, cell biology, and physiology. Accordingly, we find orthologs for only $42 \%$ of the 6623 G. sulphuraria proteins in C. merolae, and only $25 \%$ of both genomes constitute syntenic blocks (fig. S2). Coding sequences make up $77.5 \%$ of the G. sulphuraria genome, resulting in a median intergenic distance of 20 base pairs (bp) (fig. S3). Protein-coding genes contain on average two introns (fig. S4), with median lengths of $55 \mathrm{bp}$ (fig. S5). Thus, the G. sulphuraria genome is highly condensed by comparison with that of $C$. merolae and most other eukaryotes.

Eukaryotic innovations usually arise through gene duplications and neofunctionalizations, which lead to expansion of existing gene families (8). In contrast, archaea and bacteria commonly adapt through horizontal gene transfer (HGT) from other lineages (9). HGT has also been observed in some unicellular eukaryotes (10); however, to our knowledge, horizontally acquired genes have not been linked to fitness-relevant traits in freeliving eukaryotes (11). Phylogenetic analyses of G. sulphuraria genes using highly stringent criteria indicate at least 75 separate gene acquisitions from archaea and bacteria (supplementary materials). The origin of these G. sulphuraria genes from HGT is supported by the finding that compared to the genomic average, they have

${ }^{1}$ Department of Botany, Oklahoma State University, Stillwater, OK 74078, USA. ${ }^{2}$ Institute of Plant Biochemistry, HeinrichHeine-Universität Düsseldorf, 40225 Düsseldorf, Germany. ${ }^{3}$ Institute for Computer Science, Heinrich-Heine-Universität Düsseldorf, 40225 Düsseldorf, Germany. ${ }^{4}$ European Molecular Biology Laboratory (EMBL) Heidelberg, EMBL, Meyerhofstrasse 1, 69117 Heidelberg, Germany. ${ }^{5}$ Department of Plant Biology, 612 Wilson Road, Michigan State University, East Lansing, MI 48824, USA. ${ }^{6}$ Institut für Mathematik und Informatik, Ernst Moritz Arndt Universität Greifswald, Walther-Rathenau-Straße 47, 17487 Greifswald, Germany. ${ }^{7}$ Department of Earth and Environmental Sciences, 4011 CC Little Building, 1100 North University Avenue, University of Michigan, Ann Arbor, MI 48109 USA. ${ }^{8}$ Department of Earth and Planetary Science, Department of Environmental Science, Policy, and Management, University of California, Berkeley CA 94720-4767, USA. 'Department of Biochemistry and Molecular Biology, 603 Wilson Road, Michigan State University, East Lansing, MI 48824, USA. ${ }^{10}$ Research Technology Support Facility, Plant Biology Laboratories, 612 Wilson Road, Michigan State University, East Lansing, MI 48824, USA. ${ }^{11}$ Faculty of Biology and BIOSS Centre for Biological Signalling Studies, University of Freiburg, Schänzlestrasse 1, 79104 Freiburg, Germany. ${ }^{12}$ UMR USTL-INRA 1281 "Stress Abiotiques et Différenciation des Végétaux cultivés," Université de Lille 1, 59650 Villeneuve d'Ascq Cédex, France. ${ }^{13}$ Department of Microbiology and Molecular Genetics, Oklahoma State University, Stillwater, OK 74078, USA. ${ }^{14}$ CyanoBiofuels GmbH, Magnusstrasse 11, 12489 Berlin, Germany. ${ }^{15}$ Cluster of Excellence on Plant Sciences (CEPLAS), Heinrich-Heine-Universität Düsseldorf, 40225 Düsseldorf, Germany.

*To whom correspondence should be addressed. E-mail: gerald.schoenknecht@okstate.edu (G.S.); andreas.weber@ uni-duesseldorf.de (A.P.M.W.)

†These authors contributed equally to this work.

‡Permanent address: Novozymes, Inc, 1445 Drew Avenue, Davis, CA 95618, USA.

§Permanent address: Scripps Institution of Oceanography, University of California, San Diego, CA 92037, USA.

IIPermanent address: Faculty of Biology, Philipps-University Marburg, 35032 Marburg, Germany. 
significantly fewer introns (mean 0.8 versus 2.06 , $P=0.0012$, Mann-Whitney test; fig. S6), slightly higher GC content $(40.6 \%$ versus $39.9 \%, P=$ 0.0030 , Student's $t$ test; fig. S7), and deviating oligonucleotide usage ( $P=0.00034$, Mann-Whitney test; fig. S7). Gene transfers can be traced to a broad range of donor taxa (fig. S8 and table S4), with a significant enrichment from extremophile bacteria $\left(P=7.8 \times 10^{-9}\right.$, Fisher's exact test). The genome of $G$. sulphuraria thus shows notable contributions from a pan-domain gene pool.

The two largest $G$. sulphuraria protein families form a monophyletic branch within the so-called archaeal ATPases (adenosine triphosphatases) (Fig. 2A). These soluble ATPases have not been observed in other eukaryotes. Phylogenetic analyses indicate that $G$. sulphuraria acquired an ancestral ATPase gene from archaea, followed by duplications and diversification into separate families (fig. S11). Genes encoding the different families cluster in pairs on the G. sulphuraria genome (Fig. 2B). Pairs of homologous ATPase genes that are transcribed together are observed in archaeal genomes (12). Although their physiological function is unknown (13), archaeal ATPases may contribute to heat tolerance. We found a cor-

Fig. 1. Photoautotrophic (left) and heterotrophic (right) G. sulphuraria cells. Cell cultures (top) and light microscopic images (bottom; bar represents $10 \mu \mathrm{m}$ ) of $G$. sulphuraria cells grown under continuous illumination in the absence of glucose (left) or in darkness in the presence of $200 \mathrm{mM}$ glucose (right).

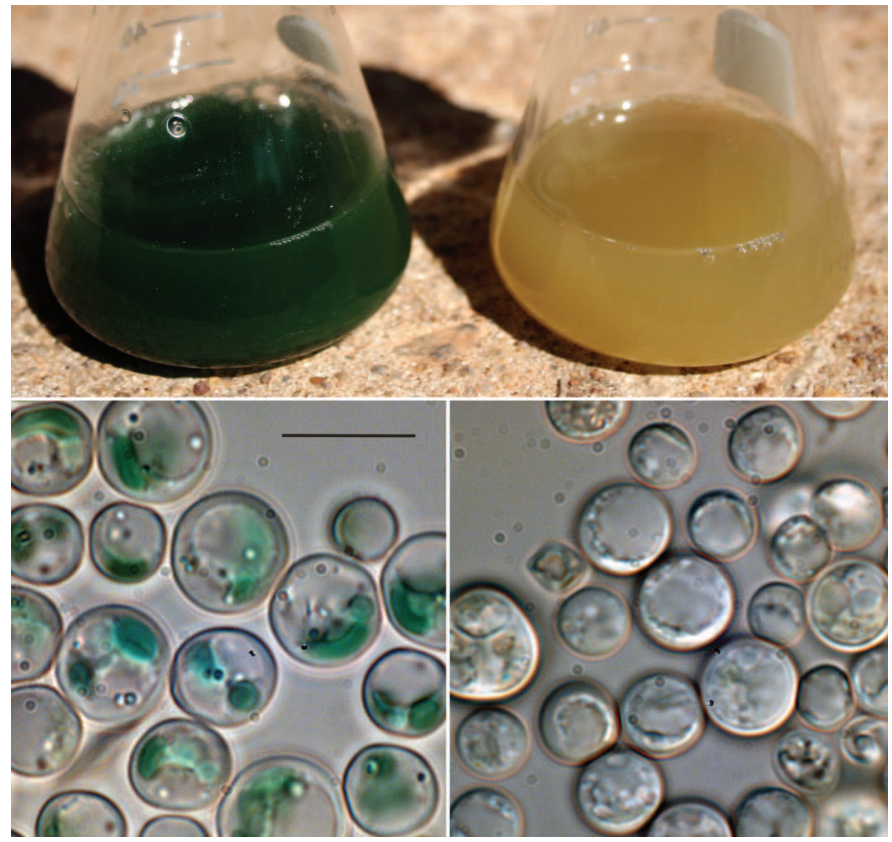

relation between ATPase gene copy number and optimal growth temperature across thermophilic and hyperthermophilic archaea (Fig. 2C). These findings suggest that $G$. sulphuraria's adaptation to heat may have been facilitated by the acquisition and subsequent expansion of an archaeal ATPase gene family.

G. sulphuraria's tolerance to high salinity is also likely to have been facilitated by HGT. Resistance to salt stress requires the removal of $\mathrm{Na}^{+}$ from the cytosol and an increase of osmolarity using compatible solutes in the cytosol. In addition to several $\mathrm{Na}^{+}: \mathrm{H}^{+}$antiporters of eukaryotic origin, G. sulphuraria encodes two monovalent cation:proton antiporters that appear to have been acquired from bacteria (fig. S13). Furthermore, genes encoding sarcosine dimethylglycine methyltransferase (SDMT) appear to originate from halophilic cyanobacteria (fig. S14). These enzymes allow the production of the compatible solute betaine from glycine (14), which indeed accumulates in $G$. sulphuraria under salt stress (fig. S15).

How does G. sulphuraria maintain near-neutral cytosolic $\mathrm{pH}$ against a $10^{6}$-fold $\mathrm{H}^{+}$gradient across its plasma membrane $(15,16)$ ? There is no evidence for an enhanced capacity to pump $\mathrm{H}^{+}$out of the cytosol (which would be an energetically intense strategy). Yet, there are indications of a reduced $\mathrm{H}^{+}$permeability of the plasma membrane. For G. sulphuraria, one voltage-gated ion channel gene was identified in the genome, compared to three in C. merolae (table S3) and 16 or more in other unicellular algae. Voltage-gated ion channels allow single-file diffusion of water, and therefore have a very high conductance for protons. A plasma membrane devoid of protonconducting voltage-gated ion channels probably
A

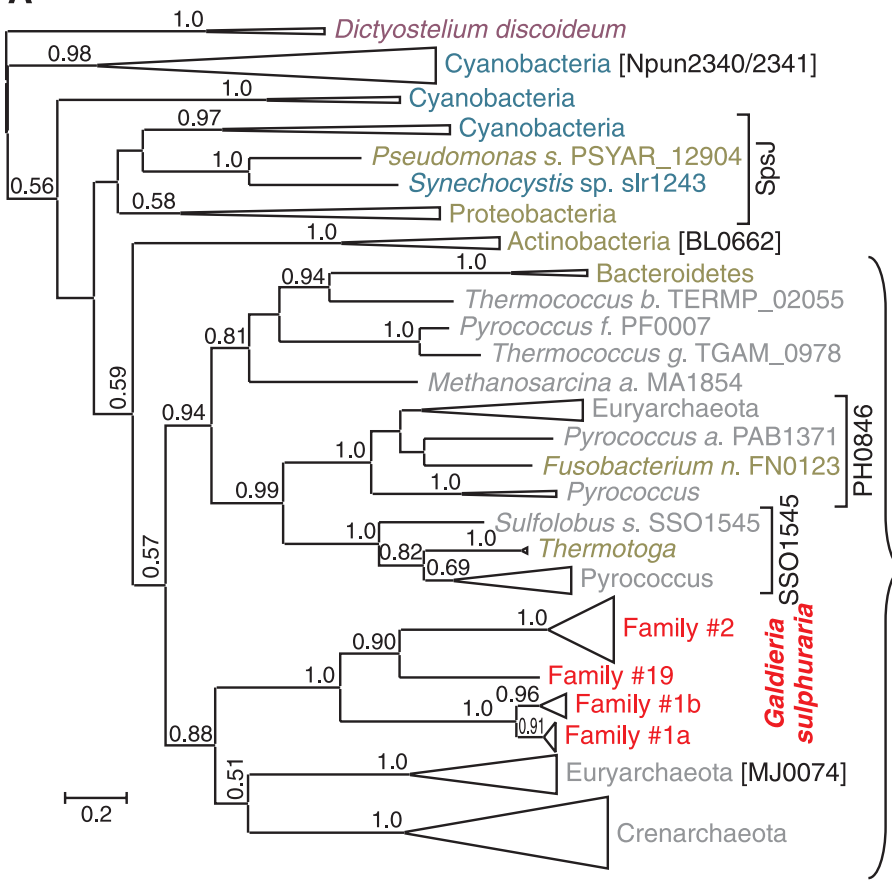

B
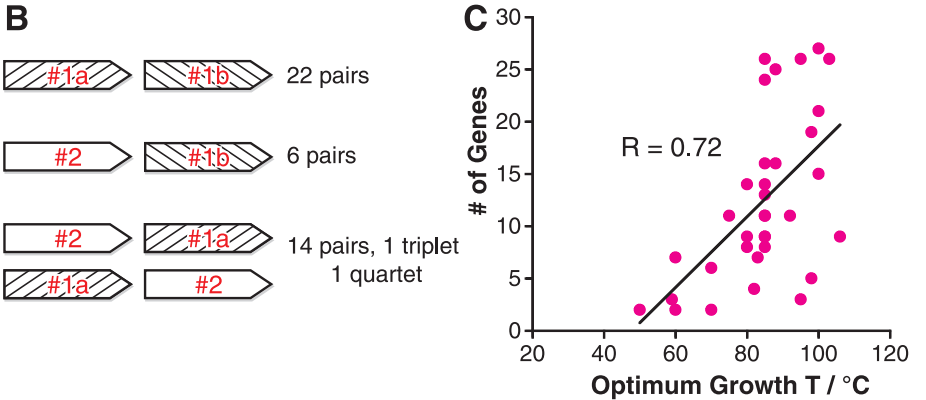

Fig. 2. Archaeal ATPases in G. sulphuraria. (A) The phylogeny of archaeal ATPases indicates HGT into G. sulphuraria. The unrooted Bayesian tree is shown with posterior probabilities (full tree and details in fig. S9, alignment of ATPase domains in fig. S10, and detailed tree of families \#1a, $\# 1$ b, \#2, and \#19 in fig. S11). Major phylogenetic groups are color-coded (Archaea, gray; Cyanobacteria, teal; other Bacteria, olive; Rhodophyta, red; Amoebozoa, purple) and labeled according to Leipe et al. (20). (B) Genes encoding archaeal ATPases in G. sulphuraria form unidirectional clusters in specific combinations; pairs are always formed from different (sub)families, with \#1b genes always at the $5^{\prime}$ end. (C) Copy number of archaeal ATPase genes in genomes of thermophilic and hyperthermophilic archaea is correlated with optimum growth temperature (for details, see fig. S12). 
has a low $\mathrm{H}^{+}$permeability, preventing the acidification of the cell interior even at very high external $\mathrm{H}^{+}$concentrations. G. sulphuraria shows an expansion of both, the intracellular chloride channel (CLIC) family and the chloride carrier/ channel (ClC) family (table S3), which do not conduct protons.

G. sulphuraria copes with toxic metals typically found in volcanic areas and acid mine drainage by a variety of metal transporters (table S3). Different plasma membrane uptake systems for divalent metal cations permit the selective uptake of essential metals (such as iron or copper) at high concentrations of toxic metals (such as aluminum or cadmium). G. sulphuraria can also neutralize biohazardous metals, making it potentially useful in biotechnological applications. For example, G. sulphuraria arsenite methyltransferases can biotransform arsenic, which is often found at very high concentrations in geothermal environments, into less toxic and possibly gaseous methyl derivatives (17). In addition, two intronless G. sulphuraria genes encode the bacterial arsenical membrane protein pump, ArsB. The sequences most similar to G. sulphuraria ArsB are from thermoacidophilic bacteria (Fig. 3), again indicating a central role for HGT in extremophilic adaptation. Mercury is found at concentrations up to $200 \mu \mathrm{g} / \mathrm{g}$ in soils from which $G$. sulphuraria has been isolated. G. sulphuraria can reduce cytotoxic $\mathrm{Hg}^{2+}$ into less toxic metallic mercury. The enzyme responsible, mercuric reductase, was also most likely acquired horizontally from Proteobacteria (fig. S17).

In total, $5.2 \%$ of $G$. sulphuraria genes encode membrane transport proteins (mostly metabolite transporters), which is more than has been discovered in most other eukaryotes (fig. S18). Gene family expansions (table S3) are found, for example, in sugar porters (fig. S19), amino acid/auxin permeases (fig. S20), and putative acetate transporters (fig. S21); these three protein families are among the 20 largest in G. sulphuraria (table S2). Further major expansions were found in amino acid-polyamine-organocation transporters (fig. S22), glycoside-pentoside-hexuronide:cation

symporters, and glycerol uptake facilitators (fig. S23). Together, these metabolite transporters are likely to be essential for the exceptional ability of G. sulphuraria to grow heterotrophically on many different metabolites. In contrast, most other unicellular algae, including C. merolae, are strictly (or almost strictly) photoautotrophic.

Again, phylogenetic analyses indicate that HGT contributed to G. sulphuraria's enormous metabolic flexibility. All acetate permeases (fig. S21) appear to originate from bacteria, whereas some of the amino acid-polyamine-organocation transporters (fig. S22) seem to stem from thermoacidophilic archaea. G. sulphuraria can grow heterotrophically on glycerol as sole carbon source (3) using a family of five glycerol uptake facilitators and a family of three glycerol dehydrogenases; both families apparently originate from HGT (figs. S23 and S24).

Some of G. sulphuraria's metabolic pathways appear to be conserved from a heterotrophic last common eukaryotic ancestor, but subsequently were lost from other photosynthetic eukaryotes. For example, animals (metazoa) use the methylmalonyl-coenzyme A pathway for the degradation of odd-numbered chain fatty acids and leucine. Whereas green plants and C. merolae lack this pathway, it is present in G. sulphuraria, as well as in diatoms and brown algae. Furthermore, green plants, diatoms, and brown algae synthesize the essential nicotinamide adenine dinucleotide precursor quinolinate from aspartate. In contrast, G. sulphuraria and C. merolae produce quinolinate from tryptophan by way of kynurenine, a pathway common to animals and fungi.

G. sulphuraria contains a number of metabolite transporter families that group with fungi and show less similarity to metabolite transporter families from more closely related organisms (figs. S19, S20, and S25). This unexpected phylogenetic trace could be explained by the loss of genes present in the common eukaryotic ancestor from other clades, by HGT from unsequenced bacteria or archaea into both eukaryotic lineages, or by HGT between fungi and G. sulphuraria. We

Fig. 3. The phylogeny (Bayesian tree) of bacterial arsenical resistance efflux pumps (ArsB) indicates HGT into $G$. sulphuraria (full tree and details in fig. S16). The curly bracket marks G. sulphuraria and four thermophilic and/or acidophilic bacteria (in olive), which live in the same environment as G. sulphuraria and from which taxa the algal ArsB may have derived.

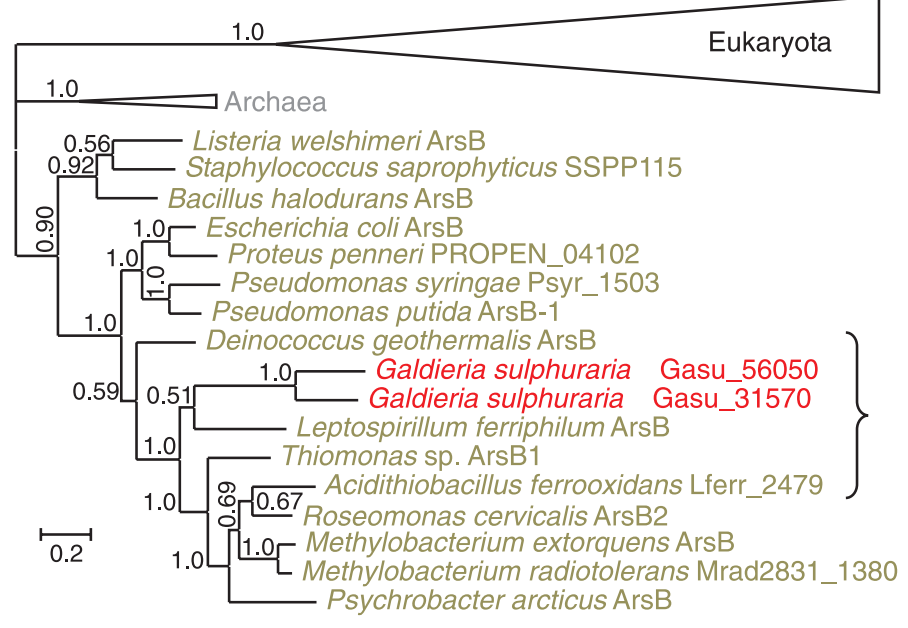

currently cannot distinguish between these alternative hypotheses owing to limited taxon representation in sequence databases.

G. sulphuraria can also survive saprophytically by excreting catabolic enzymes that decompose extracellular organic polymers into small metabolites for uptake by plasma membrane transporters. Proteomics (18) and/or bioinformatics analyses (supplementary materials) indicate excretion of aspartyl proteases, which cleave peptide bonds at acidic $\mathrm{pH} ; \beta$-galactosidases (fig. S26) and glucoamylases, which degrade polysaccharides; and acid phosphatases (fig. S27), which remove phosphate groups from organic molecules. These excreted enzymes lack orthologs in other photosynthetic eukaryotes, but show homology to excreted enzymes encoded by fungi or bacteria. In particular, G. sulphuraria may have acquired acid phosphatases (fig. S27) and some $\beta$-galactosidases (fig. S26) from bacteria.

Extensive gene transfer appears to have been key to the genomic evolution of a metabolically versatile, extremophilic, red alga. Numerous proteins acquired through HGT interact with G. sulphuraria's physico-chemical and metabolic environment. Protein families acquired horizontally by $G$. sulphuraria are 3-fold enriched in membrane transporters $(10.5 \%$ for HGT families, $P=0.010$, Fisher's exact test) and 14-fold enriched in protein families also found in extremophilic bacteria or archaea $(86.8 \%$ for HGT families, $P=1.5 \times 10^{-22}$ ). These findings for G. sulphuraria mirror the results of a previous systematic study, which showed that proteobacterial adaptation relies on the horizontal acquisition of genes that function at the bacteria's interface to the environment (19). Whereas the importance of HGT for evolution of Bacteria and Archaea is well established, adaptation of a eukaryotic extremophile by gene transfer from Bacteria and Archaea is unexpected and shines a new light on the evolution of unicellular eukaryotes.

References and Notes

1. W. N. Doemel, T. D. Brock, J. Gen. Microbiol. 67, 17 (1971).

2. L. ]. Rothschild, R. L. Mancinelli, Nature 409, 1092 (2001).

3. W. Gross, C. Schnarrenberger, Plant Cell Physiol. 36, 633 (1995).

4. V. Reeb, D. Bhattacharya, in Red Algae in the Genomic Age, J. Seckbach, D. J. Chapman, Eds. (Springer, Netherlands, 2010), pp. 409-426.

5. G. Pinto, C. Ciniglia, C. Cascone, A. Pollio, in Algae and Cyanobacteria in Extreme Environments, ]. Seckbach, Ed. (Springer, Dordrecht, Netherlands, 2007), vol. 11, pp. 487-502.

6. Materials and methods are available as supplementary materials on Science Online.

7. M. Matsuzaki et al., Nature 428, 653 (2004).

8. H. Innan, F. Kondrashov, Nat. Rev. Genet. 11, 97 (2010)

9. T. J. Treangen, E. P. C. Rocha, PLoS Genet. 7, e1001284 (2011).

10. C. Bowler et al., Nature 456, 239 (2008).

11. P. J. Keeling, Curr. Opin. Genet. Dev. 19, 613 (2009). 
12. R. Overbeek et al., Nucleic Acids Res. 33, 5691 (2005).

13. E. V. Koonin, Science 275, 1489 (1997).

14. J. G. McCoy et al., Proteins Struct. Funct. Bioinformatics 74, 368 (2009).

15. I. Enami, H. Akutsu, Y. Kyogoku, Plant Cell Physiol. 27 , 1351 (1986).

16. W. Gross, Hydrobiologia 433, 31 (2000).

17. J. Ye, C. Rensing, B. P. Rosen, Y.-G. Zhu, Trends Plant Sci. 17, 155 (2012)

18. C. Oesterhelt, S. Vogelbein, R. P. Shrestha, M. Stanke, A. P. M. Weber, Planta 227, 353 (2008)

19. C. Pál, B. Papp, M. J. Lercher, Nat. Genet. 37, 1372 (2005).

20. D. D. Leipe, E. V. Koonin, L. Aravind, J. Mol. Biol. 343, 1 (2004).
Acknowledgments: This work was made possible by NSF grant EF 0332882 (to A.P.M.W.). Partial support came from the Deutsche Forschungsgemeinschaft (DFG) (CRC TR1, IRTG 1525/1 and WE2231/7-1 to A.P.M.W., EXC 1028 to M.J.L. and A.P.M.W., CRC 680 to M.J.L., and a DFG Mercator Fellowship to G.S.). G.S. appreciates support from NSF (MCB 0925298) and the College of Arts and Sciences, Oklahoma State University (OSU). We thank M. Hanikenne and E. Koonin for helpful discussion, and A. Doust and P. Pelser for advice on phylogenetic analyses. We are grateful to B. Sears for introduction to and assistance with $\mathrm{CsCl}$ purification of bisbenzamide-treated nuclear DNA. Some of the computing for this project was performed at the OSU High Performance Computing Center. Sequence data have been deposited at DNA Data Bank of Japan, EMBL, and GenBank under accession ADNM00000000 (SRA012465). The version described in this paper is the first version, ADNM01000000.

Supplementary Materials

www.sciencemag.org/cgi/content/full/339/6124/1207/DC1

Materials and Methods

Supplementary Text

Figs. S1 to S27

Tables S1 to S3

Additional Data Table 54

References (21-79)

18 October 2012; accepted 8 January 2013

10.1126/science. 1231707

\section{Cell Death from Antibiotics Without the Involvement of Reactive Oxygen Species}

\section{Yuanyuan Liu and James A. Imlay*}

Recent observations have suggested that classic antibiotics kill bacteria by stimulating the formation of reactive oxygen species (ROS). If true, this notion might guide new strategies to improve antibiotic efficacy. In this study, the model was directly tested. Contrary to the hypothesis, antibiotic treatment did not accelerate the formation of hydrogen peroxide in Escherichia coli and did not elevate intracellular free iron, an essential reactant for the production of lethal damage. Lethality persisted in the absence of oxygen, and DNA repair mutants were not hypersensitive, undermining the idea that toxicity arose from oxidative DNA lesions. We conclude that these antibiotic exposures did not produce ROS and that lethality more likely resulted from the direct inhibition of cell-wall assembly, protein synthesis, and DNA replication.

$\mathrm{I}$ recent decades, the growing number of antibiotic-resistant pathogens has spurred efforts to further understand and improve the efficacy of the basic antibiotic classes. Most clinically used antibiotics target cell-wall assembly, protein synthesis, or DNA replication. However, recent reports have raised the possibility that although these antibiotics block growth by directly inhibiting the targets mentioned above, they may owe their lethal effects to the indirect creation of reactive oxygen species (ROS) that then damage bacterial DNA (1-10).

The evidence supporting this proposal included the observation that cell-penetrating dyes were oxidized more quickly inside antibiotic-treated bacteria (3-8). Furthermore, iron chelators $(3,4,7-9)$, which suppress hydroxyl-radical-generating Fenton chemistry, and thiourea $(4,6,8-10)$, a potential scavenger of hydroxyl radicals, lessened toxicity. Mutations that diminish fluxes through the tricarboxylic acid cycle were protective (3-5), suggesting a key role for respiration, and DNA-repair mutants were somewhat sensitive $(4,8)$. Systems analysis of aminoglycoside-treated Escherichia coli suggested a model that fits these data (5). It

Department of Microbiology, University of Illinois, Urbana, IL 61801, USA.

*To whom correspondence should be addressed. E-mail: jimlay@illinois.edu was postulated that interference with ribosome progression would release incomplete polypeptides, some of which are translocated to the cell membranes where they might trigger envelope stress. The Arc regulatory system is perturbed, potentially accelerating respiration and thereby increasing the flux of superoxide and hydrogen peroxide into the cell interior. These two oxidants

Fig. 1. Antibiotic efficacy does not require oxygen or $\mathrm{H}_{2} \mathrm{O}_{2}$. (A to C) Wild-type cells were treated with ampicillin $(A)$, norfloxacin (B), or kanamycin (C) in the presence (solid squares) or absence (open squares) of oxygen. In (C), anoxic killing was also tested in the presence of $40 \mathrm{mM} \mathrm{KNO}_{3}$ (gray squares). (D to F) Wildtype cells (solid squares, MG1655) and congenic $\mathrm{Hpx}^{-}$cells (open diamonds, AL427) were treated with antibiotics ampicillin (D), norfloxacin (E), or kanamycin (F). Results are representative of at least three biological replicates. have known sequelae that ultimately lead to DNA damage. Specifically, superoxide and hydrogen peroxide damage the iron-sulfur clusters of dehydratases $(11,12)$, releasing iron atoms and elevating the pool of intracellular unincorporated iron $(13,14)$. This iron can then react with hydrogen peroxide in the Fenton reaction, generating hydroxyl radicals that either directly damage DNA (15) or indirectly oxidize the deoxynucleotide pool, which is subsequently incorporated into DNA (10). This scenario could explain the observed oxidation of intracellular dyes, protection by scavengers and chelators, a requirement for respiration, and the sensitivity of DNA-repair mutants.

This model is plausible, so we devised experiments to directly test the molecular events that underpin it. The bacterial strain (E. coli MG1655), growth medium (LB), and antibiotic doses were chosen to match those of previous studies (4). Kanamycin was used to target translation, ampicillin to block cell-wall synthesis, and norfloxacin to disrupt DNA replication.

Superoxide and hydrogen peroxide are generated inside cells when flavoenzymes inadvertently transfer a fraction of their electron flux directly to molecular oxygen $(15,17)$. Thus, neither of these ROS can be formed under anoxic
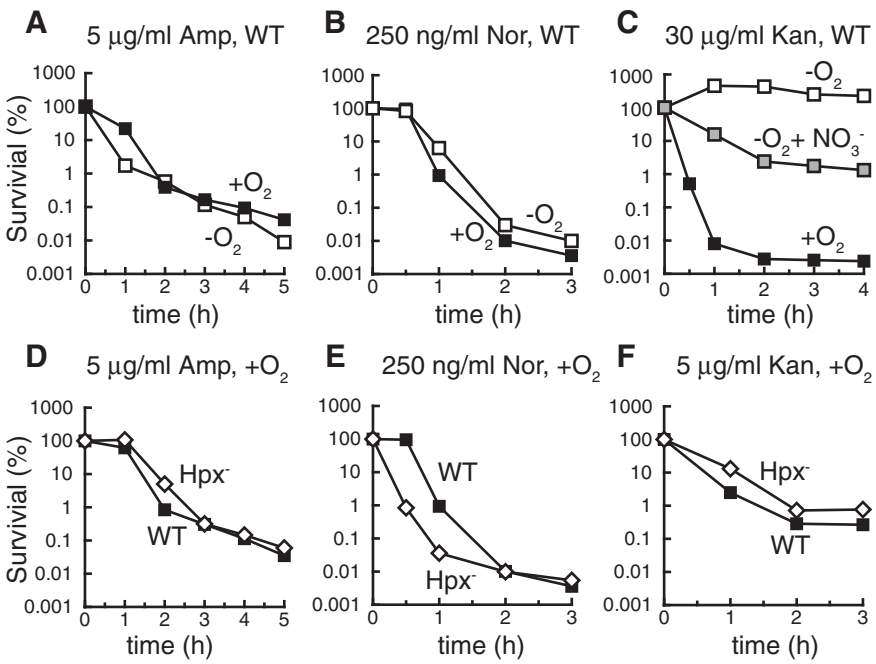


\section{Science}

\section{Gene Transfer from Bacteria and Archaea Facilitated Evolution of an Extremophilic Eukaryote}

Gerald Schönknecht, Wei-Hua Chen, Chad M. Ternes, Guillaume G. Barbier, Roshan P. Shrestha, Mario Stanke, Andrea Bräutigam, Brett J. Baker, Jillian F. Banfield, R. Michael Garavito, Kevin Carr, Curtis Wilkerson, Stefan A. Rensing, David Gagneul, Nicholas E. Dickenson, Christine Oesterhelt, Martin J. Lercher and Andreas P. M. Weber

Science 339 (6124), 1207-1210.

DOI: $10.1126 /$ science. 1231707

\section{Hot, Toxic Eukaryote}

Unusually, the single-celled eukaryote red alga, Galdieria sulphuraria, can thrive in hot, acidic springs. This organism is endowed with extraordinary metabolic talents and can consume a variety of strange carbohydrates, as well as turn on photosynthesis when the food runs out. Schönknecht et al. (p. 1207; see the Perspective by Rocha) discerned from phylogenetic analysis of its genome that during its evolution, G. sulphuraria appears to have commandeered at least 75 bacterial and archaeal genes by horizontal gene transfer and then applied gene expansion to boost its metabolic repertoire.

ARTICLE TOOLS

SUPPLEMENTARY MATERIALS

RELATED CONTENT

REFERENCES

PERMISSIONS http://science.sciencemag.org/content/339/6124/1207

http://science.sciencemag.org/content/suppl/2013/03/07/339.6124.1207.DC1

http://science.sciencemag.org/content/sci/339/6124/1154.full

This article cites 72 articles, 10 of which you can access for free http://science.sciencemag.org/content/339/6124/1207\#BIBL

http://www.sciencemag.org/help/reprints-and-permissions

Use of this article is subject to the Terms of Service

Science (print ISSN 0036-8075; online ISSN 1095-9203) is published by the American Association for the Advancement of Science, 1200 New York Avenue NW, Washington, DC 20005. 2017 () The Authors, some rights reserved; exclusive licensee American Association for the Advancement of Science. No claim to original U.S. Government Works. The title Science is a registered trademark of AAAS. 